
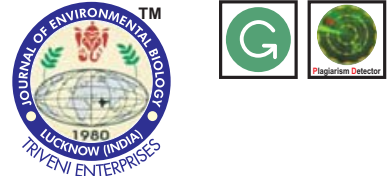

DOI: https://doi.org/10.22438/jeb/38/3/MS-201
Journal of Environmental Biology

\section{Genetic diversity and population structure analysis of Chrysanthemum (Dendranthema grandiflora Tzvelev) germplasm based on RAPD markers}

Abstract

Authors Info

S. Kumar ${ }^{1 *}$, M. Kumar', H. K. Yadav', S. Sharma ${ }^{3}$ and S. Kumar

${ }^{1}$ Department of Horticulture, Sardar Vallabhbhai Patel University of Agriculture and Technology, Meerut-250 110, India

${ }^{2}$ Genetics and Molecular Biology Division, CSIR-National Botanical Research Institute, Lucknow-226 001, India

${ }^{3}$ Department of Crop Science, Institute of Genomic Biology, University of Illinois,

Urbana-Champaign-61801, USA

*Corresponding Author Email : drskflori@yahoo.com

Key words

Chrysanthemum,

Cluster,

Genetic diversity,

Population structure,

RAPD

Publication Info

Paper received: 04.01 .2016

Revised received: 13.03 .2016

Re-revised received : 15.07 .2016

Accepted: 05.08 .2016
Aim: The present investigation was carried out to characterize the genetic variation present in a set of 38 Indian chrysanthemum cultivars using RAPD (random amplified polymorphic DNA) markers. Unlike to previous molecular markers based diversity studies, the present study involved population structure analysis which revealed subpopulations and admixtures in set of chrysanthemum cultivars studied.

Methodology: The experimental material of the present investigation comprised of 38 chrysanthemum cultivars collected from different part of India. Total genomic DNA was extracted from fresh and young leaf tissues following CTAB method. Polymorphic information content and resolving power of primers were calculated to select useful primers. Dendrogram and PCoA analysis were carried out to reveal the genetic diversity and relatedness among cultivars. Population structure analysis was carried out to decipher any subpopulations and admixtures in the chrysanthemum germplasm.

Results: A total of 70 clear and scorable bands were produced by 10 RAPD primers. Out of 70 bands, 66 were polymorphic and four were monomorphic. All the 38 cultivars were grouped into 2 sub-population. Mixed population ancestry was observed among the two clusters.

Interpretation: Sufficient diversity was observed among the germplasm. Results of present study will be helpful for future population and selective breeding studies targeting overall chrysanthemum improvement. Diverse cultivars identified in the present study can be utilized in multi-parent breeding program to develop varieties with desirable traits.

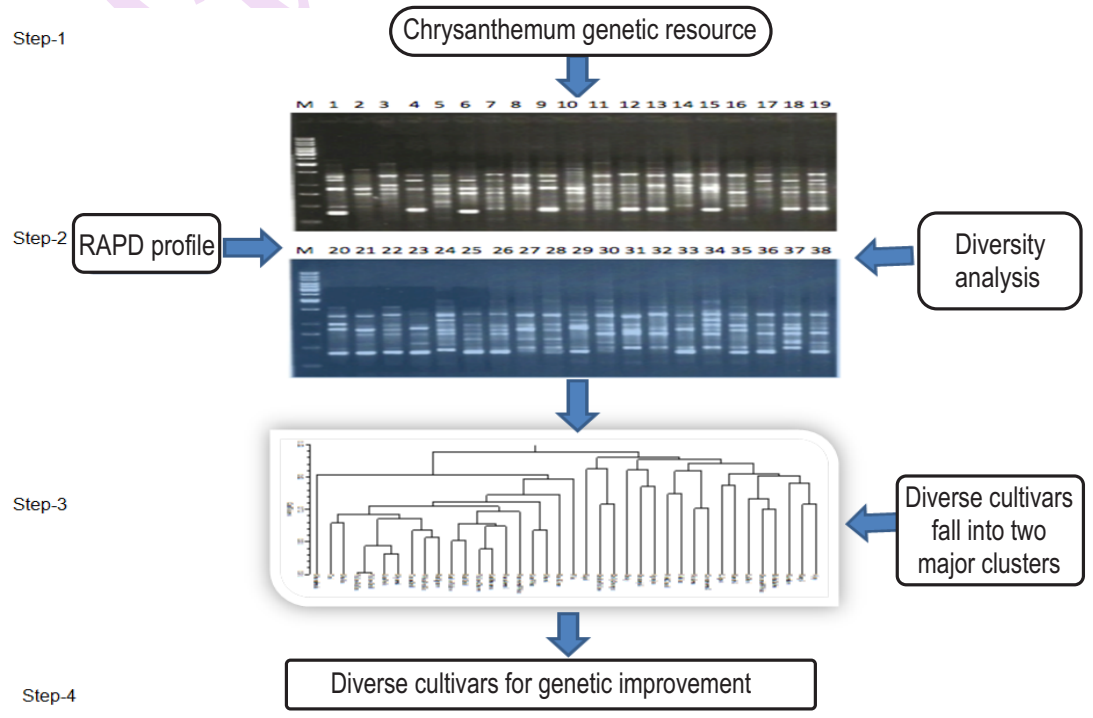




\section{Introduction}

Chrysanthemum (Dendranthema grandiflora Tzvelev) is one of the major cut flower crops in domestic and international market that rank second after rose. It is commonly known by names like Guldaudi, Queen of the East and Glory of the East (Randhawa and Mukhopadhyay, 2001). Most of the cultivated species have basic chromosome number of $9(x)$ with wide range of ploidy level $(2 n=36-75)$. Most of them are allo-hexaploid and aneuploid having most frequent somatic chromosome number of 54 (Kher, 1988; Zhang et al., 2013). The evaluation of chrysanthemum remained controversial so far; however, it is generally believed that the species is a hybrid complex derived from chance hybridization that naturally occurred between species of Chrysanthemum vestitum, Chrysanthemum indicum, Chrysanthemum lavandulifolium and Chrysanthemum zawadskii (Tang et al., 2009, 2011; Wang et al., 2004). The species has a strong self-incompatibility system like other members of Asteraceae (Li and Chen, 2007).

Because of its wide occurrence and high economic importance intense breeding programs are underway worldwide. Large germplasm resources has been maintained for this purpose. The number of chrysanthemum cultivars available worldwide is about 20,000 to 30,000 (Anderson, 2006). Beside decoration, some species of chrysanthemum are also used the production of essential oil (C. morifolium), insecticides ( $C$. coccineum, C. cinerariifolium) and culinary items (Bose et al., 2002).

In recent times, several studies have been conducted on chrysanthemum involving different aspects like cytology (El-Twab and Kondo, 2012; Zhang et al., 2013), morphology (Banerji et al., 2012; Hong et al., 2012), physiology (Leiss et al., 2009), and functional genomics (Chen et al., 2012; Gu et al., 2011). Unfortunately, very few molecular genetic studies have been conducted in chrysenthemum (Baliyan et al., 2014; Zhang and Wang, 2013). This can be attributed to aneuploid polyploidy nature of the chrysanthemum genome that involves loss or gain of chromosomes due to hybridization and mutation of chrysanthemum cultivars (Zhang and Wang, 2013). Therefore, it is important to evaluate and characterize the genetic diversity among chrysanthemum cultivars for crop improvement and conservation. Morphological and molecular markers have become prerequisite of germplasm management, conservation, improvement, IPR protection and DUS testing. Diversity analysis is important for deciphering genetic relationship including parentage and for the efficient management of germplasm (Mukherjee et al., 2013, Baliyan et al., 2014 and Kumar et al., 2015). In light of the above, present investigation was undertaken to characterize genetic variation and population structure present in a subset of 38 Indian chrysanthemum cultivars by random amplified polymorphic DNA markers. Unlike to previous RAPD and other molecular markers based genetic diversity studies, present study involves population structure analysis to decipher any subpopulations and admixtures in different set of chrysanthemum cultivars. Results of the present study will facilitate selective breeding in chrysanthemum.

\section{Materials and Methods}

Plant materials : The experimental material of the present investigation comprised of 38 chrysanthemum cultivars collected from different part of India (Table 1).

DNA extraction : Total genomic DNA was extracted from fresh and young leaf tissues following CTAB method (Doyle and Doyle, 1990). DNA concentration was determined using a Bio-Rad's Smart Spec ${ }^{\mathrm{TM}}$ Plus spectrophotometer.

RAPD analysis : For initial screening 30 RAPD primers (Integrated DNA Technologies) were used. Ten primers, which showed good amplification, were used for subsequent fingerprinting analysis. Details of these primers is provided in Table 2. PCR amplification was carried out in $25 \mu \mathrm{l}$ reaction volume containing $50 \mathrm{ng}$ DNA, 1x PCR buffer, 10 pmole primer, $200 \mu \mathrm{M}$ dNTPs, $2 \mathrm{mM} \mathrm{MgCl}$ and $1 \mathrm{U}$ Taq polymerase. The amplification reactions were performed in PTC Thermal Cycler (MJ Research Inc.) with following conditions: $5 \mathrm{~min}$ initial denaturation at $94^{\circ} \mathrm{C}, 40$ cycles of denaturation at $94^{\circ} \mathrm{C}$ for $1 \mathrm{~min}$, annealing at $36-38{ }^{\circ} \mathrm{C}$ (according to primers annealing temperature) and 2 min primer extension at $72{ }^{\circ} \mathrm{C}$ and final extension at $72{ }^{\circ} \mathrm{C}$ for $8 \mathrm{~min}$. The amplified PCR product was resolved on $2.0 \%$ agarose gel in $1 \times$ TBE buffer. The bands were visualized on a UV transilluminator and photographed by gel documentation system. The bands were scored manually as 1 (presence) and 0 (absence).

Data analysis : Allelic polymorphic information content (PIC) was calculated for each primer following Botstein et al. (1980). Resolving Power (Rp) was measured by Prevost and Wilkinson's (1999). The 0-1 matrix data of RAPD analysis was subjected to calculate pairwise genetic similarity using Jaccard's coefficient (Jaccard, 1908). The similarity matrix thus obtained was subjected to prepare dendrogram using unweighted pair group method of arithmetic averages (UPGMA) with the help of NTSYSPC software version 2.02e (Rohlf, 1993). Principal coordinate analysis $(\mathrm{PCo})$ was also performed using similarity matrix. Further, in order to estimate the number of subpopulations in the chrysanthemum germplasm, population STRUCTURE analysis was done using program STRUCTURE version 2.2 (Pritchard et al., 2000). The membership of each cultivars was tested for $K=2$ to $K=10$ with admixture model. Three independent runs were assessed for each fixed $K$ and each run consisted of 30,000 burnin period and 1,00,000 iterations. The optimal value of $K$ was determined by examining $\Delta \mathrm{K}$ statistic and $\mathrm{L}(\mathrm{K})$ (Evanno et al., 2005) using Structure Harvester program (Earl and von Holdt, 2012). 


\section{Results and Discussion}

Determination of genetic variation present in individuals or population is a prerequisite for any breeding study. Similarly, characterization of germplasm structure is necessary for conservation, management and association studies (Odong et al., 2011; Zeinalabedini et al., 2012; Roein et al., 2014). In chrysanthemum, complex genome, high level of heterozygosity and self incompatibility makes genetic improvement difficult (Anderson and Ascher 2000; Anderson 2006; Zhang et al., 2010, 2011). Recently, various types of molecular markers AFLP (Roein et al., 2014), SSRs (Zhang and Wang, 2013), SRAP (Shao et al., 2010; Zhang et al., 2011a, b), ISSRs (Shao et al., 2010; Baliyan et al., 2014) and RAPD (Sehrawat et al. 2003) have been used to decipher the genetic diversity in chrysanthemum. Out of these, AFLP, SSR and SRAP markers generate more informative data points. However, cost involved in their development and subsequent application limits the usage of these useful markers. Further, usage of co-dominant markers like SSR is rather difficult in chrysanthemums because of complicated genetic background due to hexaploid-based aneuploid nature which makes the data scoring and further analysis tedious (Zhang et al., 2013). In this scenario, ISSRs and RAPDs can be cost effective markers alternate with potential of providing enough useful genetic data points.

Present study helped in understanding the genetic relatedness at DNA level among the 38 cultivars of chrysanthemum. A total of 70 clear and scorable bands were produced by 10 RAPD primers. Out of 70 bands, 66 were polymorphic and four were monomorphic. Polymorphism level ranged from 66.66 to $100 \%$ (Table 2). Maximum number of bands

Table 1 : List of Indian Chrysanthemum cultivars used for diversity analysis

\begin{tabular}{llll}
\hline S.No./ld. No. & Chrysanthemum cultivars & S.No./ld. No. & Chrysanthemum cultivars \\
\hline 1 & Ajay & 20 & Poornima White \\
2 & Aparjita & 21 & PusaAnmol \\
3 & Birbal Sahni & 22 & Ravi Kiran \\
4 & Corcon Small & 23 & Red Gold \\
5 & Dolly Orange & 24 & Red Spoon \\
6 & Flirt & 25 & Sadbhavana \\
7 & Gaity & 26 & Sadvin Yellow \\
8 & Geetanjali & 27 & Shanti \\
9 & Golden Yellow & 28 & Sharadmala \\
10 & Greenish White & 29 & Shukla \\
11 & Jubilee & 30 & Shyamal \\
12 & Kajol & 31 & Star Pink \\
13 & Kanchil & 32 & Star White \\
14 & Kargil & 33 & Teri \\
15 & Kundan & 34 & Texas Gold \\
16 & LalPari & 35 & White Prolific \\
17 & Liliput & 36 & Yellow Charm \\
18 & Neelima & 37 & Yellow Gold \\
19 & PinkCloud & 38 & Yellow Reflex \\
\hline
\end{tabular}

Table 2 : Details of RAPD banding pattern used for the fingerprinting of 38 Chrysenthemum cultivars

\begin{tabular}{|c|c|c|c|c|c|c|c|}
\hline \multirow[t]{2}{*}{ Primer code } & \multirow[t]{2}{*}{ Primer sequence } & \multirow{2}{*}{$\begin{array}{l}\text { Average Band/ } \\
\text { Primer }\end{array}$} & \multicolumn{2}{|c|}{ Number of bands } & \multirow{2}{*}{$\begin{array}{l}\text { Polymorphism } \\
\%\end{array}$} & \multirow{2}{*}{$\begin{array}{l}\text { PIC } \\
\text { Value }\end{array}$} & \multirow{2}{*}{$\begin{array}{l}\text { Resolving } \\
\text { Power }\end{array}$} \\
\hline & & & Poly-morphic & Mono-morphic & & & \\
\hline OPX-16 & 5'CTCTGTTCGG3' & 6.47 & 10 & 1 & $90.9 \%$ & 0.5738 & 11.72 \\
\hline OPJ-08 & 5'CATACCGTGG3' & 5.47 & 6 & 0 & $100 \%$ & 0.391 & 7.72 \\
\hline OPF-06 & 5'GGGAATTCGG3' & 6.5 & 8 & 1 & $88.88 \%$ & 0.565 & 10.46 \\
\hline OPC-07 & 5'GTCCCGACGA3' & 3.5 & 4 & 0 & $100 \%$ & 0.445 & 5.4 \\
\hline OPD-08 & 5'GTGTGCCCCA3' & 2.71 & 3 & 0 & $100 \%$ & 0.524 & 3.78 \\
\hline OPK-11 & 5'AATGCCCCAG3' & 6.34 & 11 & 0 & $100 \%$ & 0.625 & 12.33 \\
\hline OPF-13 & 5'GGCTGCAGAA3' & 4.97 & 6 & 0 & $100 \%$ & 0.425 & 7.52 \\
\hline OPF-14 & 5'TGCTGCAGGT3' & 7.25 & 8 & 0 & $100 \%$ & 0.404 & 12.2 \\
\hline OPC-15 & 5'GACGGATCAG3' & 5.26 & 4 & 2 & $66.66 \%$ & 0.207 & 2.33 \\
\hline \multirow[t]{2}{*}{ OPF-17 } & 5'AACCCGGGAA3' & 4.92 & 6 & 0 & $100 \%$ & 0.546 & 10.52 \\
\hline & Total & 66 & 4 & 946.44 & 4.705 & 83.98 & \\
\hline Average & & & & 94.644 & 0.4705 & 8.398 & \\
\hline
\end{tabular}




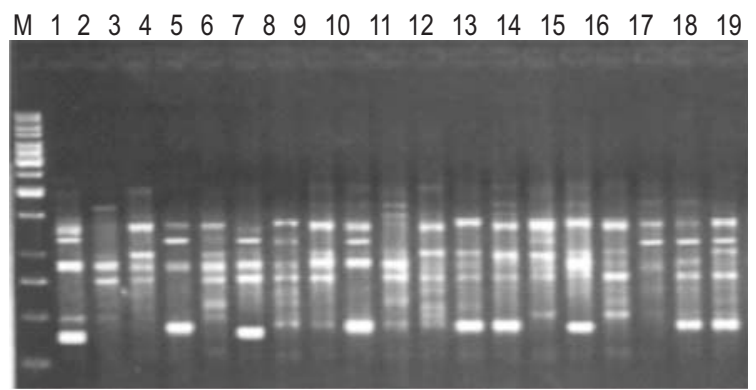

M $2021222324252627282930 \quad 313233 \quad 343536 \quad 3738$

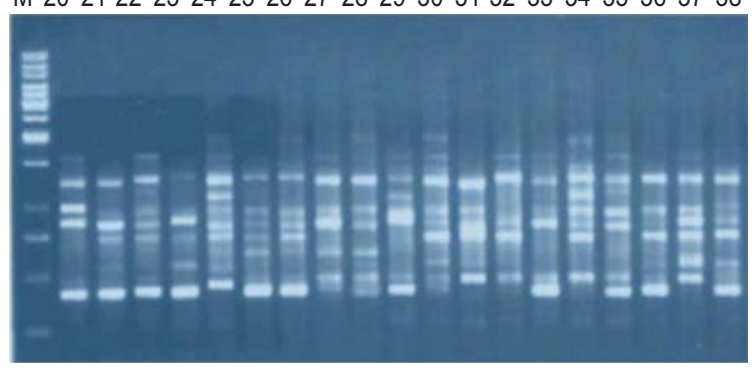

Fig. 1 : RAPD band pattern of 38 Chrysanthemum cultivars by primer OPK-11. M represents marker ladder

were obtained with primers OPK-11 (11). The PIC value ranged from 0.207 (OPC-15) to 0.625 (OPK-11) with an average of 0.470 . The resolving power of RAPD primer varied from 2.33 (OPC-15) to 12.33 (OPK-11) with an average of 8.398. Thus, RAPD primer OPK-11 was most polymorphic (Fig. 1). The pairwise Jaccard's coefficient of genetic similarity varied from 0.41 to 0.90 with an average of $0.59 \pm 0.08$. The UPGMA based clustering grouped all the cultivars, into two major clusters namely cluster I and II with each two subclusters namely la, b and Ila, b (Fig. 2). Cluster I had 18 cultivars while cluster II consisted of 19 cultivars. Maximum similarity was noticed between Yellow Gold and Yellow Reflex (0.90) cultivars, however, minimum similarity was noticed between Sharadmala and Aparajita (0.41) followed by Pusa Anmol and Ajay (0.41), Pusa Anmol and Golden Yellow (0.41). The cultivar Sharadmala did not clustere with other cultivars. Similar to UPGMA based dendrogram, Principal coordinate analysis $(\mathrm{PCOA})$ also divided 38 chrysanthemum cultivars into two main clusters with cultivar Sharadmala present between two clusters (Fig. 3). Mukherjee et al. (2013) also performed RAPD analysis of a set of 40 elite varieties of chrysanthemum and reported average similarity coefficient of 0.42 and all were grouped into two clusters. Chatterjee et al. (2006) found high genetic distance among different chrysanthemum and reported that there exists a possibility of introgressing new and novel genes from the chrysanthemum gene pool. Kumar et al. (2006) also revealed that RAPD molecular markers can be used to assess polymorphism among the mutants and suggested that these markers may be useful for plant variety protection in future. Sehrawat et al. (2003) reported that RAPDs are efficient for identification of chrysanthemum cultivars and determination of genetic relationship. Similar to the results obtained in the present study, Sehrawat et al. (2003) classified 13 chrysanthemum cultivars into two main groups with RAPD markers. Authors also found that the results were consistent with the morphological differences and geographical distributions (Sehrawat et al., 2003). Similarly, in other ornamentals such as orchid, RAPD marker based diversity was assessed among 20 orchid hybrids and reported existence of considerable genetic variability (Thomas et al., 2010). Liu and Yang (2010) evaluated 10 wild and 12 cultivars of chrysanthemum using RAPD markers and reported $96 \%$ polymorphism. The AFLP based genetic diversity among 65 cultivars of chrysanthemum showed $73 \%$ marker polymorphism and significant variability among the cultivars (Wu etal., 2007).

Unlike to previous RAPD based studies, present study involves population STRUCTURE analysis which is helpful to know the presence of subpopulations and admixtures. This model based clustering by STRUCTURE analysis gives information about origin and admixture of alleles among the cultivars used in the study. STRUCTURE program was used to estimate the number of genetically distinct populations $(\mathrm{K})$ and admixtures. Value of $\mathrm{K}=2$ was chosen for final analysis of population structure as the highest value of $\mathrm{K}$ among the 38 genotypes was $\mathrm{K}=2$ based on the Bayesian clustering of information from the 10 RAPD primer combinations (Fig. 4). At $\mathrm{K}=2$, all the 38 cultivars were grouped into 2 sub-population. Mixed population ancestry was observed among the two clusters. The cultivar Neelima, Jubilee, Flirt in sub population 1 and Sharadmala, Ravi Kiran in subpopulation 2 showed to some extent of admixturing (Fig. 5). Admixtures have been also observed in a recent AFLP based diversity study on 48 chrysanthemum genotypes from Iran (Roein et al., 2014). These mixed populations may be attributed to domestication history, breeding, resource exchange, high heterozygosity and self-incompatibility of Chrysanthemums (Roein et al., 2014; Anderson 2006; Zhang et al., 2010; Zhao et al., 2010). However, number of admixtures observed by Roein et al. (2014) in Iranian chrysanthemum germaplasm is much more in comparison to that observed in present study suggesting possible intense breeding effort in former case. In the present study, clustering based on the STRUCTURE analysis was also found to be in congruence with the distance based clustering as shown in dendrogram and $\mathrm{PCoA}$ analysis.

The genetic similarities obtained from the present analysis can be used for selection of cultivars as parents to generate mapping populations for breeding purposes. Results of the present study suggested that cultivars namely, Aparajita, Gaity, Flirt, Pusa Anmol, Ajay and Golden Yellow to be highly diverse at molecular level and could be used in developing mapping population and for tagging QTLs for important ornamental traits as demonstrated in recent genetic mapping 


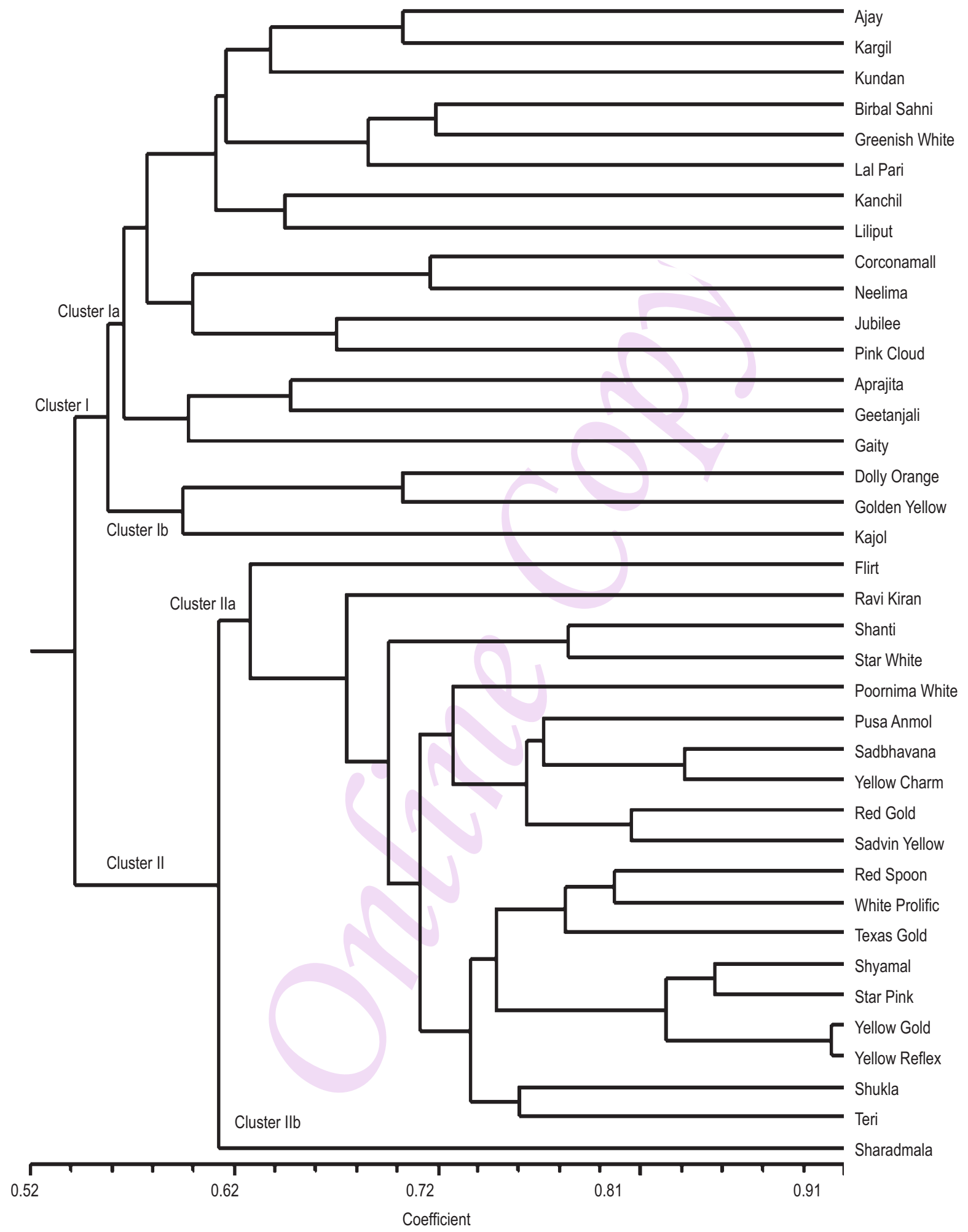

Fig. 2 : Dendrogram of 38 cultivars of Chrysanthemum based on Jaccards similarity coefficient and UPGMA clustering 


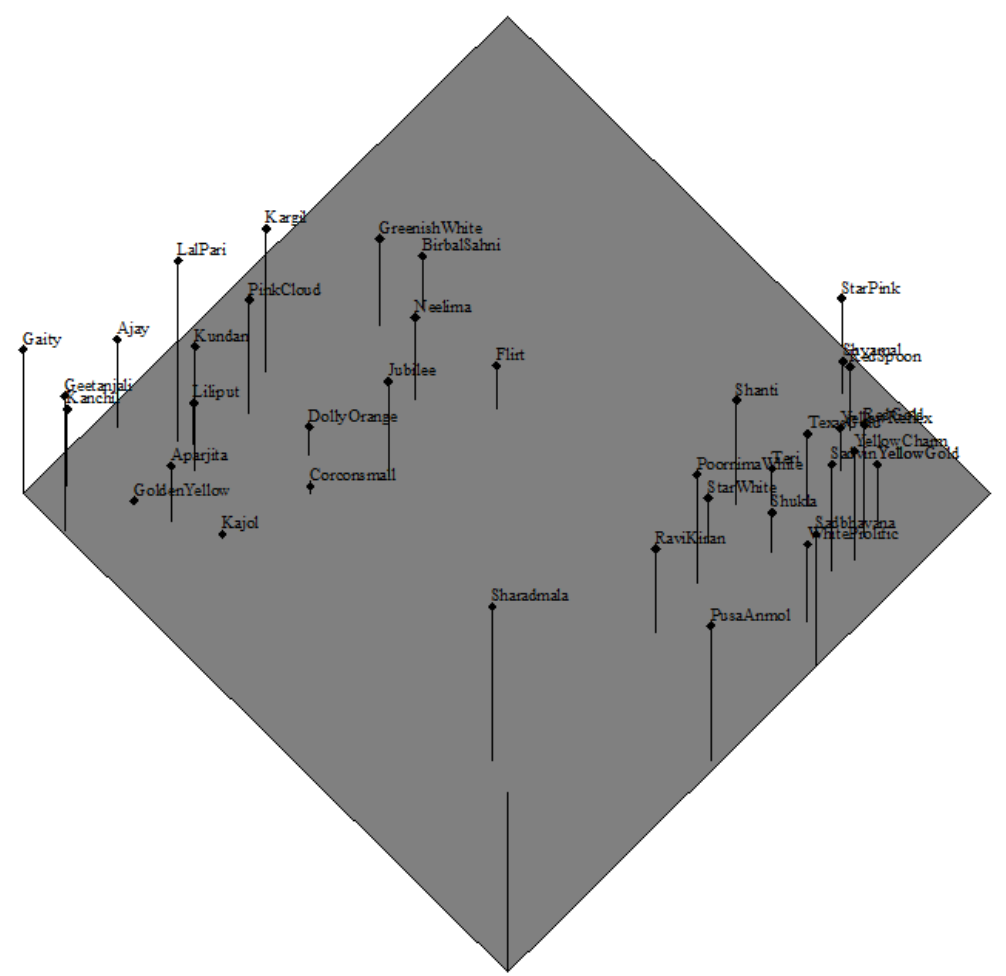

Fig. 3 : Grouping pattern of 38 cultivars of Chrysanthemum by Principal Coordinate Analysis (PCoA)

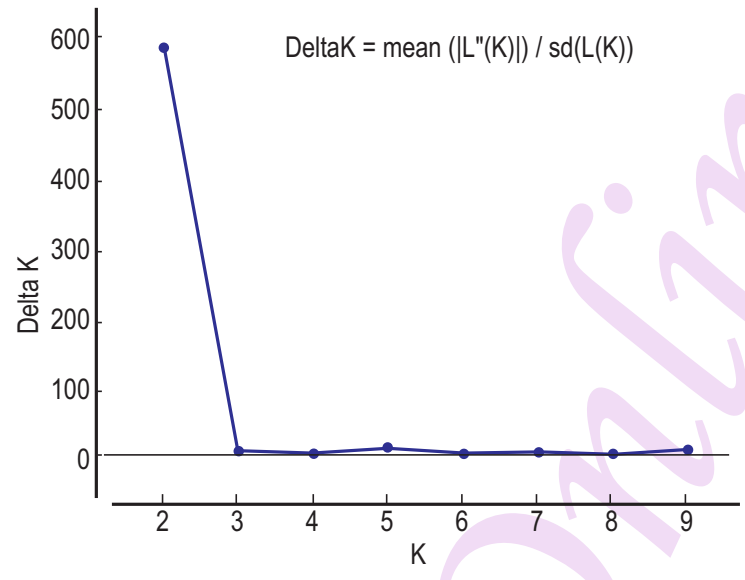

Fig. 4 : Graphical plot of Delta Kvalues studies in chrysanthemum (Zhang et al., 2010; 2011a,b; 2013). Present RAPD and other molecular marker (AFLPs, SSRs, ISSRs and SRAP) based diversity studies indicated that the ample genetic diversity or variations was present in the chrysanthemum germplasm with polymorphic loci ranging from $66.0 \%$ to $100.0 \%$. However, it is noteworthy that sample size in all of the genetic diversity studies conducted in chrysanthemum, including the present one, was insufficient (ranging from only 12 to 48 plants) that could not cover the entire genetic background of the chrysanthemum. Therefore, it is highly desirable to increase the sample size in order to capture all of the hidden genetic diversity in chrysanthemum germplasm. In near future, development of high-throughput single nucleotide polymnorphism (SNP) markers would be highly useful for indepth study of chrysanthemum germplasm. Nevertheless, results

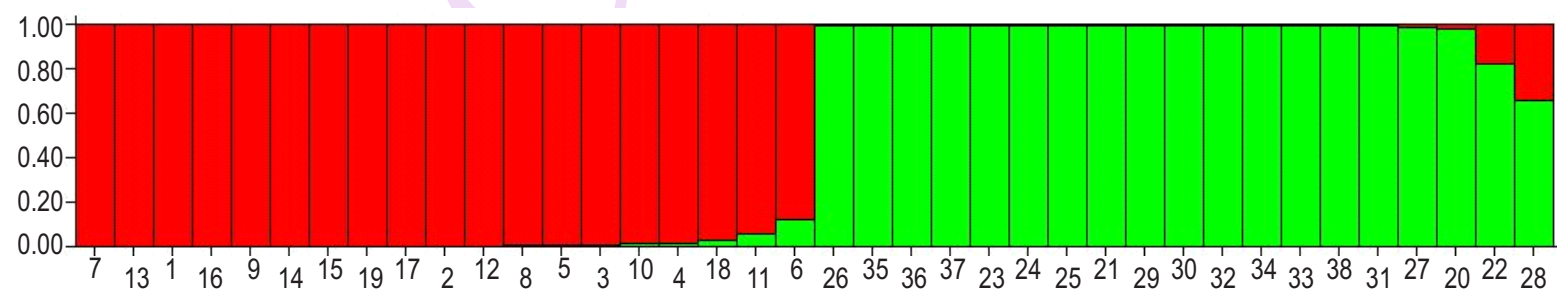

Fig. 5 : Sub population among 38 cultivars of Chrysanthemum based on structure analysis. Number on x axis corresponds to cultivars number in Table 1. 
of the present study will be helpful for future population and selective breeding studies targeting overall chrysanthemum improvement. Diverse cultivars identified in the present study can be utilized in multi-parent breeding program to develop varieties with desirable traits.

\section{Acknowledgment}

Authors thank Vice Chancellor, Sardar Vallabhbhai Patel Univeristy of Agriculture and Technology, Meerut for conceptualizing the work on Chrysanthemum and facilities provided during the investigation.

\section{References}

Anderson, N.O.: Chrysanthemum. Dendranthema grandiflora Tzvelv. In: Flower breeding and genetics: issues, challenges, and opportunities for the 21 st century (Ed.: N.O. Anderson). Springer, Dordrecht, pp. 389-437 (2006).

Anderson, N.O. and P.D. Ascher: Fertility changes in inbred families of self-incompatible chrysanthemums (Dendranthema grandiflorum). J.Am. Soc. Hortic. Sci., 125, 619-625 (2000).

Baliyan, D., A. Sirohi, M. Kumar, V. Kumar, S. Malik, S. Sharma and S. Sharma: Comparative genetic diversity analysis in chrysanthemum: A pilot study based on morpho-agronomic traits and ISSR markers. Sci. Horti., 167, 164-208 (2014).

Banerji, B.K., A. Batra and A.K. Dwivedi: Morphological and biochemical characterization of chrysanthemum. J. Hort. Sci., 7, 51-55 (2012).

Bose, T.K., L.P. Yadav, P. Pal, P. Das and V.A. Parthasarathy: Chrysanthemum. Commercial Flowers, Vol. 1, pp. 465-601 (2002).

Botstein, D., R.L. White, M. Skolnick and R.W. Davis: Construction of a genetic linkage map in man using restriction fragment length polymorphisms. Am. J. Hum. Genet., 32, 314-331(1980).

Chatterjee, J., A.K.A. Mandal, S.A. Ranade, J.A. Teixeira and S.K. Datta: Molecular systematics in Chrysanthemum $X$ grandiflorum (Ramat.) Kitamura. Sci. Horti., 110, 373-378 (2006).

Chen, F.D., P.D. Chen, W.M. Fang and H.J. Li: Cytogenetics of $F_{1}-$ hybrids between two small-headed cultivars of Dendranthema $\times$ grandiflorum and two wild Dendranthema species. Acta. Horti. Sin., 25, 308-309 (1998).

Chen, S., C. Li, X. Zhu, Y. Deng, W. Sun, L. Wang, F. Chen and Z. Zhang: The identification of flavonoids and the expression of genes of anthocyanin biosynthesis in the chrysanthemum flowers. Biol. Plant., 56,458-464 (2012).

Dai S.L., J.Y. Chen and W.B. Li: Application of RAPD analysis in the study on the origin of Chinese cultivated chrysanthemum. Acta. Bot. Sin., 40, 1053-1059 (1998).

Doyle, J.J. and J.L. Doyle: Isolation of plant DNA from fresh tissue. Focus, 12, 13-15(1990).

Earl, D.A. and B.M. von Holdt: STRUCTURE HARVESTER: A website and program for visualizing STRUCTURE output and implementing the Evanno method. Conserv. Genet. Resour., 4, 359-361(2012).

El-Twab, M.H. and K. Kondo: Physical mapping of $5 \mathrm{~S}$ and $45 \mathrm{~S}$ rDNA in Chrysanthemum and related genera of the Anthemideae by FISH, and species relationships. J. Genet., 91,245-249 (2012).

Evanno, G., S. Regnaut and J. Goudet: Detecting the number of clusters of individuals using the software STRUCTURE: A simulation study. Mol. Ecol., 14, 2611-2620 (2005).

Gu, C., S. Chen, Z. Liu, H. Shan, H. Luo, Z. Guan, and F. Chen. Reference gene selection for quantitative real-time PCR in chrysanthemum subjected to biotic and abiotic stress. Mol. Biotechnol., 49,192-197 (2011).

Hong, Y., X. Bai, W. Sun, F. Jia and S. Dai: The numerical classification of chrysanthemum flower color phenotype. Hort. Sinica, 39,1330-1340 (2012).

Jaccard, P.: Nouvelles recherches sur la distribution florale. Bull. Soc. Vaud. Nat., 44, 223-270 (1908).

Kher, M.A.: Chrysanthemum in India. Associated Publishers Co., New Delhi, p. 79 (1988).

Kumar, J., K.P. Singh, K.V. Prasad, N. Verma., Namita and S. Panwar: Characterization of chrysanthemum (Chrysanthemum grandiflorum) varieties using ISSR markers. Indian J. Ag. Sci., 85, 566-570 (2015)

Kumar, S., K.V. Prasad and M.L. Choudhary: Detection of genetic variability among chrysanthemum radio mutants using RAPD markers. Curr. Sci, 90, 1108-1113 (2006).

Leiss, K.A., F. Maltese, Y.H. Choi, R. Verpoorte and P.G. Klinkhamer: Identification of chlorogenic acid as a resistance factor for thrips in chrysanthemum. Plant Physiol., 15,1567-1575 (2009).

Li X.L. and F.D. Chen: Self-incompatibility in Dendranthema morifolium. J. Wuhan. Bot. Res., 25, 591-595 (2007).

Liu, R. and J.S. Yang: Genetic diversity of some Dendranthema spp. based on RAPD analysis. J. Agric. Univ. Hebei, 33, 60-65 (2010).

Mukherjee, A.K., A. Dey, L. Acharya, S.K. Palai and P.C. Panda: Studies on genetic diversity in elite varieties of chrysanthemum using RAPD and ISSR markers. Indian J. Biotech., 12, 161-112 (2013).

Odong, T.L., J. van Heerwaarden, J. Jansen, T.J.L. van Hintum, F.A. van Eeuwijk: Determination of genetic structure of germplasm collections: are traditional hierarchical clustering methods appropriate for molecular marker data? Theor. Appl. Genet. 123,195-205 (2011).

Prevost, A. and M.J. Wilkinson: A new system of comparing PCR primers applied to ISSR fingerprinting of potato accessions. Theor. Appl. Genet., 98, 107-112 (1999).

Pritchard, J.K., M. Stephens and P. Donnelly: Inference of population structure using multilocus genotype data. Genetics, 155, 945-959 (2000).

Randhawa, G.S. and A. Mukhopadhyay: Floriculture in India. Allied Publishers Limited, New Delhi, India, pp. 362-367 (2001).

Richards, A.J.: Plant Breeding Systems. George Allen and Unwin, London(1986).

Roein, Z., M.H. Asil, A. Sabouri and A.R. Dadras: Genetic structure of Chrysanthemum genotypes from Iran assessed by AFLP markers and phenotypic traits. Plant Syst. Evol., 300, 493-503 (2014).

Rohlf, F.J.: NTSYS-PC: Numerical taxonomy and multivariate analysis System. Version 2.02e, Exeter Software, Setauket, New Yark (1998).

Sehrawat, S.K., R. Kumar, D.S. Dahiya, K.S. Boora and R. Yadav: DNA fingerprinting of chrysanthemum cultivars using RAPDs. Acta. Horti., 624, 479-485 (2003).

Shao, Q., Q. Guo, Y. Deng and H. Guo. A comparative analysis of genetic diversity in medicinal Chrysanthemum morifolium based on morphology, ISSR and SRAP markers. Biochem. Syst. Ecol., 38,1160-1169 (2010).

Tang, F., F. Chen , S. Chen, N. Teng and W. Fang: Intergeneric 
hybridization and relationship of genera within the tribe Anthemideae Cass. (I. Dendranthema crassum (kitam.) kitam. $x$ Crossostephium chinense (L.) Makino). Euphytica, 169,133-140 (2009).

Tang, F., H. Wang, S. Chen, F. Chen, Z. Liu and W. Fang: Intergeneric hybridization between Dendranthema nankingense and Tanacetum vulgare. Sci. Hortic., 132, 1-6 (2011).

Thomas, B., C.L. Rani and A.T. Mathew: Molecular characterization in monopodial orchid hybrids. J. Orna. Hort., 13, $26-34$ (2010).

Wang, C., J.Y. Chen and A.J. Marten: Molecular evolution and phylogeny of florist's chrysanthemum and related species. J. Beijing Forest Univ., 1, 91-96(2004).

Wu, Z.S., H.L. Li, J.H. Liu, Z.R. Zuo and R.C. Tian: Analysis of genetic diversity among 65 chrysanthemum cultivars based on AFLP. J. Nanjing For. Univ.: Nat. Sci., 31, 67-70(2007).

Zhang, Y., M.L. Zhu and S.L. Dai: Analysis of karyotype diversity of 40 Chinese chrysanthemum cultivars. J. Syste. Evol., 51, 335-352 (2013).

Zhang, F, S. Chen, F. Chen, W. Fang and F. Li: A preliminary genetic linkage map of chrysanthemum (Chrysanthemum morifolium) cultivars using RAPD, ISSR and AFLP markers. Scientia Hort., 125,422-428(2010).

Zhang, F., S. Chen, F. Chen, W. Fang, Y. Deng, Q. Chang and P. Liu: Genetic analysis and associated SRAP markers for flowering traits of chrysanthemum (Chrysanthemum morifolium). Euphytica, 177,15-24 (2011a).

Zhang, F., S. Chen, F. Chen, W. Fang, Y. Chen and F. Li: SRAP based mapping and QTL detection for inflorescence-related traits in chrysanthemum (Chrysanthemum morifolium). Mol. Breed., 27, 11-23(2011b).

Zhang, Y. and C. Wang: Assessing the genetic diversity of chrysanthemum cultivars with microsatellites. J. Amer. Soc. Hort. Sci., 138,479-486 (2013).

Zhao, W.G., J.W. Chung, Y.I. Cho, W.H. Rha, G.A. Lee, K.H. Ma, S.H. Han, K.H. Bang, C.B. Park, S.M. Kim and Y.J. Park: Molecular genetic diversity and population structure in Lycium accessions using SSR markers. C.R. Biologies, 333,793-800 (2010).

Zeinalabedini, M., S. Sohrabi, K. Nikoumanes, A. Imani and M. Mardi: Phenotypic and molecular variability and genetic structure of Iranian almond cultivars. Plant Syst. Evol., 298,1917-1929 (2012). 